\title{
Cuatro especies nuevas del género Drosophila (Diptera, Drosophilidae) en las provincias de Pichincha, Napo y Santo Domingo de los Tsáchilas, Ecuador
}

\author{
Luz M Llangarí-Arizo (D) \& Violeta Rafael (D)
}

Laboratorio de Genética Evolutiva, Escuela de Ciencias Biológicas, Pontificia Universidad Católica del Ecuador, Apartado 17-01-2184, Quito, Ecuador. (luzmarinallangari@gmail.com; vrafael@puce.edu.ec)

Recibido 09 marzo 2018

Aceptado 11 octubre 2018

Published 29 noviembre 2018

DOI 10.1590/1678-4766e2018040

RESUMEN. Se describen cuatro especies nuevas del género Drosophila. Drosophila valenteae sp. nov., recolectada en un remanente de bosque andino de la provincia de Pichincha, pertenece al grupo D. guarani. Drosophila cumanda sp. nov., recolectada en los andes orientales de la provincia de Napo, pertenece al grupo D. annulimana. Las especies D. cruzloma sp. nov., recolectada en el páramo de la provincia de Pichincha, y D. tinalandia sp. nov., recolectadas en los andes occidentales de la provincia de Santo Domingo de los Tsáchilas, aunque pertenecen al subgénero Drosophila, no fueron asignadas a ningún grupo de especies. Sin embargo D. tinalandia sp. nov. fue capturada en inflorescencias de especies del género Xanthosoma spp. (Araceae), por lo tanto podría estar relacionada a un grupo antofílico.

PALABRAS CLAVE. Cashapamba, Cumanda, Cruz Loma, Tinalandia, valenteae.

ABSTRACT. Four new species of genus Drosophila (Diptera, Drosophilidae) in the Pichincha, Napo, and Santo Domingo de los Tsáchilas provinces, Ecuador. Four new species of the genus Drosophila are described. Drosophila valenteae sp. nov., collected in a remnant of Andean forest of the province of Pichincha, belongs to the group D. guarani. Drosophila cumanda sp. nov., collected in the eastern Andes of the province of Napo, belongs to the group $D$. annulimana. The species $D$. cruzloma sp. nov., collected in the paramo of the province of Pichincha, and $D$. tinalandia sp. nov., collected in the western Andes of the province of Santo Domingo de los Tsáchilas, although it belongs to the subgenus Drosophila, were not assigned to any group of species. However D. tinalandia sp. nov. was captured in inflorescences of species of the genus Xanthosoma spp. (Araceae), therefore it could be related to an anthophilic group.

KEYWORDS. Cashapamba, Cumanda, Cruz Loma, Tinalandia, valenteae.

La distribución de las diferentes especies del género Drosophila es muy variada, existen especies que viven exclusivamente en desiertos (Ross \& MARKow, 2006) y otras que se encuentran únicamente en los Andes (Vela \& RAFAEL, 2004).

En Ecuador han sido muestreadas 16 provincias situadas en la Costa, Sierra, Amazonía y la Región Insular. En estas regiones geográficas se han reportado la existencia de varios grupos de especies del género Drosophila (AcuRIo \& RAFAEL, 2009; CÉSPEDES \& RAFAEL, 2012). Uno de ellos es el grupo Drosophila guarani establecido por Dobzhansky \& Pavan 1943, en la actualidad está constituido por 24 especies (Bä́hLI, 2017; RATCOV et al., 2017; VAZ et al., 2018, PeÑafiel-VINUEZa \& RAFAel, 2018) y en el Ecuador se han registrado 12 especies del grupo $D$. guarani. Ellas son: Drosophila griseolineata Duda, 1927, Drosophila ornatifrons Duda, 1927, Drosophila urubamba Vilela \& Pereira, 1993, Drosophila ecuatoriana Vela \& Rafael, 2004, Drosophila pichinchana Vela \& Rafael, 2004, Drosophila quitensis Vela
\& Rafael, 2004, Drosophila cuscungu Vela \& Rafael, 2005, Drosophila zamorana, Drosophila quinarensi, Drosophila sachapuyu, Drosophila caxarumi y Drosophila misi PeñafielVinueza \& Rafael, 2018.

Otro grupo de especies presente en Ecuador es el grupo Drosophila annulimana Pavan \& Da Cunha, 1947 conformado por 18 especies (GotTSCHALK et al., 2012; CABEzas \& RAFAel, 2013), todas endémicas de la Región Neotropical (Tosi et al., 2007). En el país se han registrado cinco especies del grupo D. annulimana, ellas son: Drosophila aracataca Vilela \& Val, 1983, Drosophila araicas Pavan \& Nacrur 1950, Drosophila tarsata Schiner, 1868, Drosophila yana Vela \& Rafael, 2005, y Drosophila intillacta Cabezas \& Rafael, 2013.

En este trabajo se describen cuatro especies nuevas del subgénero Drosophila capturadas en tres provincias del Ecuador. Una de ellas pertenece al grupo $D$. guarani y la otra al grupo $D$. annulimana, las otras dos especies no fueron incluidas en ningún grupo de especies. 


\section{MATERIALES Y MÉTODOS}

Durante el año 2013 se realizaron recolecciones de drosófilas en tres provincias del Ecuador: (1) en la provincia de Pichincha, en la localidad de Sangolquí (Cashapamba, $0^{\circ} 19^{\prime} 59.3^{\prime \prime} \mathrm{S} 78^{\circ} 25^{\prime} 51^{\prime \prime} \mathrm{W}, 2417 \mathrm{msnm}$ ) y en Quito (cercanías del Teleférico, $0^{\circ} 1$ '19"'S, 78³1'25.1”'W, $3100 \mathrm{msnm}$ ); (2) en la provincia de Santo Domingo de los Tsáchilas, en Tinalandia $\left(0^{\circ} 18^{\prime} 34^{\prime \prime} \mathrm{S}, 79^{\circ} 03^{\prime} 00^{\prime \prime} \mathrm{W}, 660 \mathrm{msnm}\right.$.); (3) en la provincia de Napo, en Cumandá $\left(0^{\circ} 27^{\prime} 13.2^{\prime \prime} \mathrm{S}\right.$, $77^{\circ} 52^{\prime} 46.8^{\prime}$ 'W, $1864 \mathrm{msnm}$ ). Para la captura se utilizaron trampas de cebo de banano y levadura colocados en el interior de una botella plástica con pequeños agujeros, las moscas vivas se recolectaron con un aspirador entomológico y las moscas muertas se conservaron en alcohol. Los adultos machos sirvieron para el análisis de la genitalia e identificación de especies. Con las hembras recolectadas se fundaron isolíneas para obtener la descendencia F1, que permitió reconocer a los machos y hembras de cada especie. Los individuos recolectados se mantuvieron en medio de cultivo de banano, levadura, gelatina, limón y nipagina (RAFAEL et al., 2000) a $18^{\circ} \mathrm{C}$.

La identificación de las especies se hizo comparando la morfología externa y la genitalia con la bibliografía disponible tanto en físico como en línea, así mismo con las drosófilas conservadas en el museo. Para la descripción de las especies nuevas se utilizó la terminología propuesta por BÄCHLI et al. (2004). Las figuras fueron realizadas en un microscopio con cámara lúcida incorporada y los dibujos finales se realizaron en el programa Adobe Illustrador ${ }^{\circledR}$.

La toma de medidas para el cálculo de los índices se realizó con el programa AxioVision. Los valores entre paréntesis corresponden a las medidas de los paratipos. El holotipo, alotipo y paratipos fueron montados en seco, pegados en triángulos de cartulina con sus respectivas estructuras genitálicas colocadas en un microtubo con glicerol. Todos los individuos fueron depositados en el Museo de Zoología, sección Invertebrados de la Pontificia Universidad Católica del Ecuador, Quito (QCAZI).

\section{Grupo Drosophila guarani Drosophila valenteae sp. nov.} (Figs 1-11, 16-18)

urn:Isid:zoobank.org:act:057B1750-E6A1-41B7-91D30E4CB74E8309

Material tipo. Holotipo ô (montado en seco, genitalia en microtubo), Ecuador, Pichincha, Sangolquí (Cashapamba, 0¹9'59.3”'S, 78²5'51”W, 2417 msnm), VII.2013, V. Rafael col., L. M. Llangarí \& V. Rafael det. (QCAZI 3142); alotipo $\&$ (montado en seco, genitalia en microtubo), los mismos datos del holotipo (QCAZI 3152); paratipos: $9 \hat{\jmath}, 9+$ (montados en seco, genitalia en microtubo), mismos datos del holotipo (QCAZI 3133-3141, 3143-3151).

Diagnosis. Tórax marrón oscuro con seis hileras de pelos acrosticales entre las dorsocentrales anteriores. Cerdas escutelares basales divergentes. La vena transversal radialmedia (R-M) y la vena transversal media discal-cubital $(\mathrm{dM}-\mathrm{Cu})$ ligeramente sombreadas. Edeago recto tubular, parte distal membranosa con microproyecciones a modo de espina en el lado ventral, membranas de la parte dorsal expandidas con puntos refringentes.

Descripción del macho, holotipo y paratipos (individuos descritos en vivo, provenientes de isolínea). Morfología externa. Longitud total (cuerpo + alas) 4.05 (3.964.10) $\mathrm{mm}$. Color del cuerpo marrón.

Cabeza. Frente marrón claro. Longitud frontal $0.32(0.28-0.39) \mathrm{mm}$. Índice frontal 0.94 (0.92-0.95). Triángulo frontal marrón oscuro. Triángulo ocelar marrón claro, cerca del $37(36-38) \%$ de la longitud, ocelos amarillo oscuro. Placa orbital marrón; la cerda orbital media cerca de la orbital anterior y ligeramente hacia el borde exterior de la placa orbital, distancia de or3 a or1, 90(88-91)\% de or3 a vtm, radio or1/or3 0.76 (0.69-0.77), radio or2/or1 $0.35(0.33-0.37)$, cerda postocelar $56(54-58) \%$ y cerda ocelar $72(71-73) \%$ de la longitud frontal; índice vt 1.25 (1.23-1.29); índice vibrisal 0.70 (0.69-0.72). Frontal vitta marrón. Gena y postgena marrón claro; índice genal 4.01 (3.10-4.93). Carina marrón claro, ligeramente prominente, no surcada. Proboscis marrón claro. Ojos de color rojo vinoso, índice del ojo 1.40 (1.32-1.53). Segundo y tercer segmento antenal marrón oscuro. Arista plumosa con cinco ramas dorsales, tres ventrales, más la terminal bifurcada.

Tórax. Marrón oscuro, longitud $1.13(1.10-1.27) \mathrm{mm}$. Índice h 1.20 (1.10-1.40). Seis hileras de pelos acrosticales entre las cerdas dorsocentrales anteriores. Distancia transversal de las cerdas dorsocentrales 218 (215-221) \% de la distancia longitudinal, índice dc 0.76 (0.72-0.79). Escutelo del mismo color que el tórax, distancia entre las cerdas escutelares apicales $78(75-80) \%$ de la distancia entre las cerdas apicales y basales. Cerdas escutelares basales divergentes; índice escutelar 1.23 (1.20-1.28). Cerda esternopleural media ligeramente más pequeña que la cerda esternopleural anterior; índice esterno 0.51 (0.49-0.56). Patas amarillas.

Alas. Color beige con la vena transversal radial-media (R-M) y la vena transversal media discal-cubital (dM-Cu) ligeramente sombreadas. Longitud del ala 2.55 (2.50-2.87) $\mathrm{mm}$. Índices alares 2.21 (2.19-2.73); $\mathrm{C}=3.80$ (3.75-4.10), $\mathrm{ac}=2.00(1.84-2.20), \mathrm{hb}=0.25(0.21-0.33), 4 \mathrm{c}=0.61(0.57-0.65)$, $4 \mathrm{v}=1.47(1.36-1.54), 5 \mathrm{x}=1.34(1.28-1.36), \mathrm{M}=0.46(0.39$ $0.49)$ y prox. $x=0.43(0.38-0.50)$.

Abdomen. Marrón amarillento, con línea media dorsal. Del primer al quinto tergito con una franja oscura de forma irregular que se adelgaza hacia los lados; sexto tergito sin línea media dorsal y en el centro con una mancha oscura en forma de trapecio.

Genitalia externa. Cerci libres, parte baja del epandrio con seis cerdas, lóbulo inferior del epandrio con dos cerdas, parte dorsal microtricosa sin cerdas. Surestilo no microtricoso, con ocho (7-8) dientes primarios; lado derecho con cuatro dientes secundarios (4-5) y lado izquierdo con cinco dientes secundarios (4-5) y ocho cerdas marginales (6-8) (Fig. 1). 
1
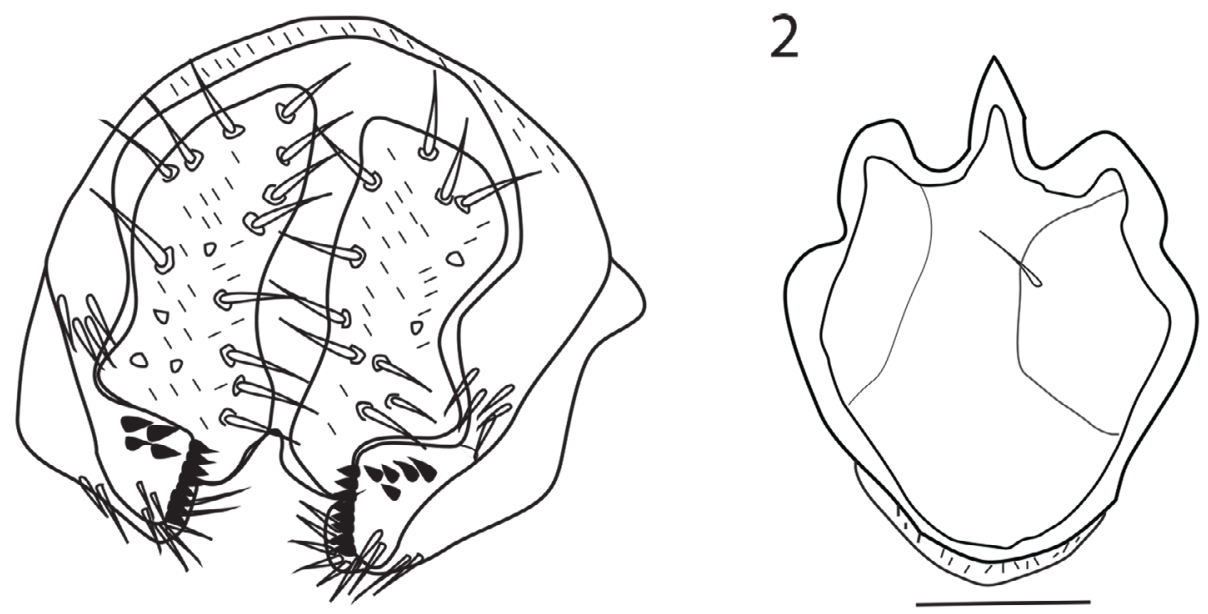

3
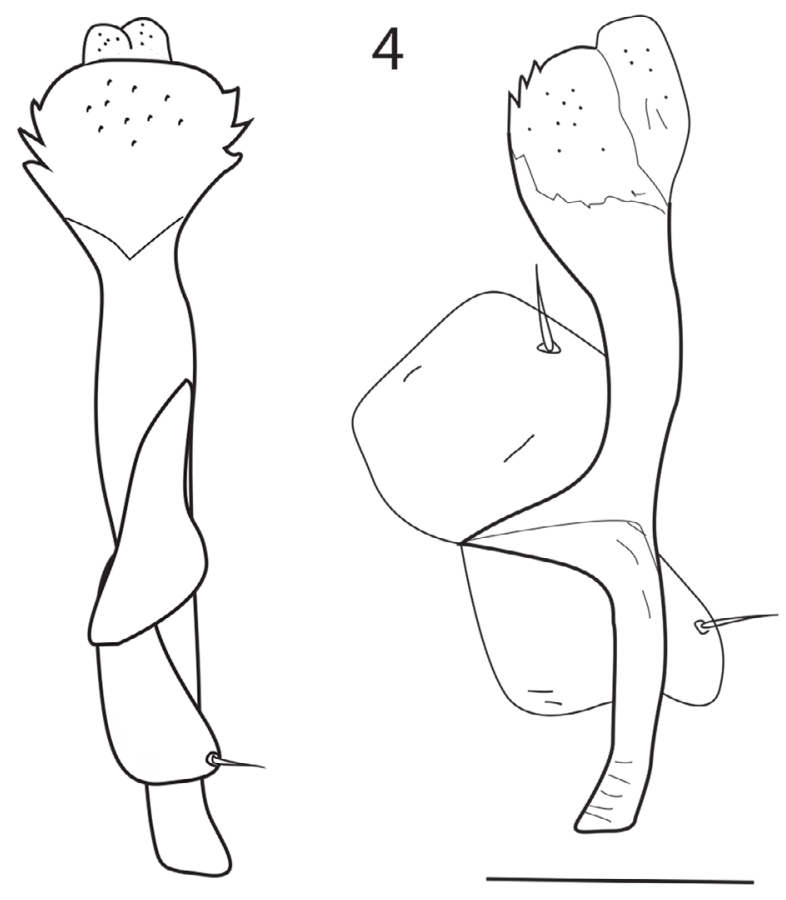

5

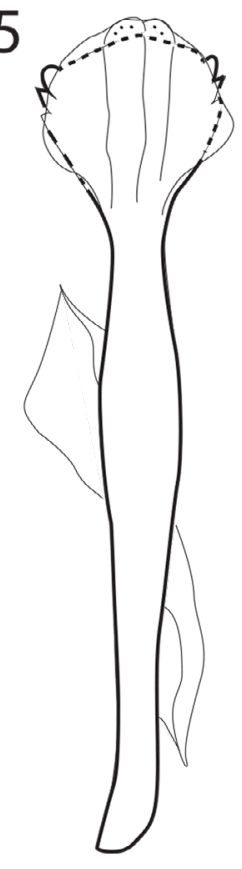

6

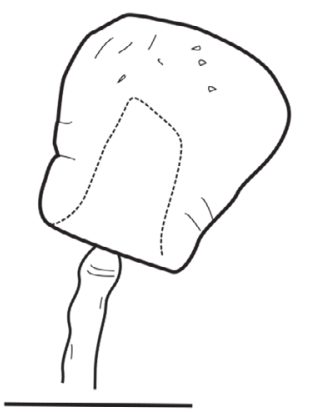

7

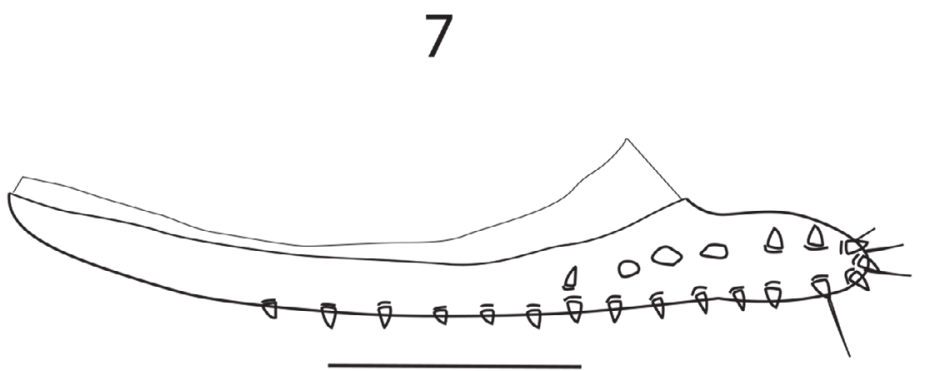

Figs 1-7. Drosophila valenteae sp. nov., holotipo $\widehat{\jmath}$ : 1, epandrio; 2 , hipandrio y gonopodio; $3-5$, edeago y paráfisis, vistas ventral, lateral y dorsal, respectivamente. Alotipo + : 6, espermateca; 7, oviscapto. (Escala: $0.1 \mathrm{~mm}$ ). 
Genitalia interna. Hipandrio en forma de escudo con el contorno quitinizado, gonopodio poco quitinizado con una cerda, arco dorsal sostenido por dos formaciones quitinosas (Fig. 2). Edeago recto tubular, parte distal expandida, con tres dientes a cada lado y microproyecciones, parte dorsal con una lámina membranosa con puntos refringentes. Paráfisis unida al gonopodio, con una cerda (Figs 3-5, 16 y 17).

Descripción de la hembra, alotipo y paratipos (individuos descritos en vivo, proveniente de isolínea).
Morfología externa. Longitud total (cuerpo + alas) 4.10 (4.054.35) mm. Color del cuerpo marrón (Fig. 8).

Medidas hembra Cabeza. Longitud frontal 0.37 (0.35$0.41) \mathrm{mm}$. Índice frontal 0.84 (0.81-0.89). Triángulo ocelar, cerca del 40.50(37.20-42.30)\% de la longitud frontal. Distancia de or3 a or1, 75\%(72-82)\% de or3 a vtm, radio or1/or3 0.92 (0.87-0.95), radio or2/or1 0.30 (0.27-0.33); cerda postocelar $57(54-60) \%$ y cerda ocelar $72(71-73) \%$ de la longitud frontal; índice vt 1.25 (1.23-1.29); índice vibrisal
8

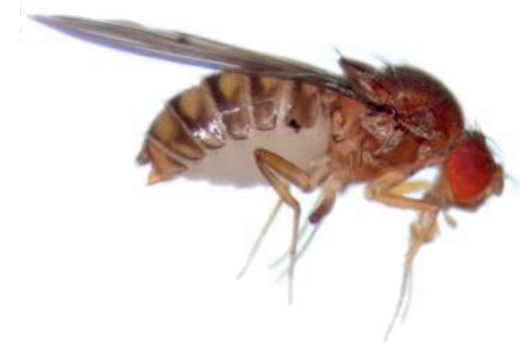

9

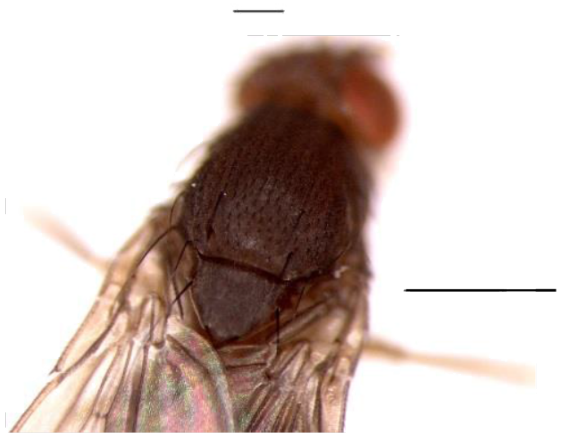

10

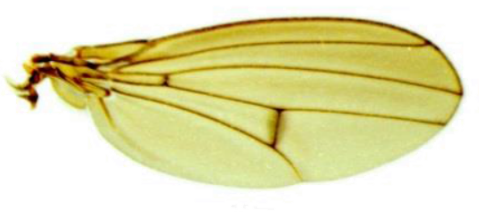

11

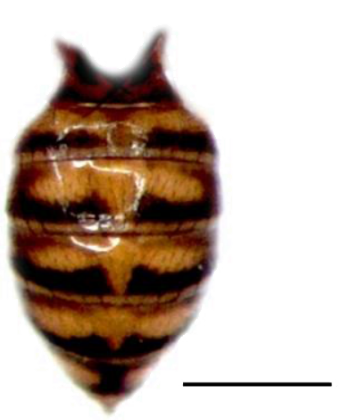

12

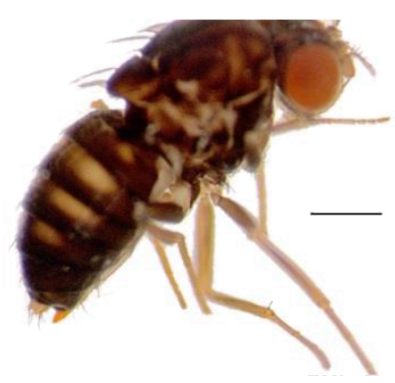

13

\section{4}
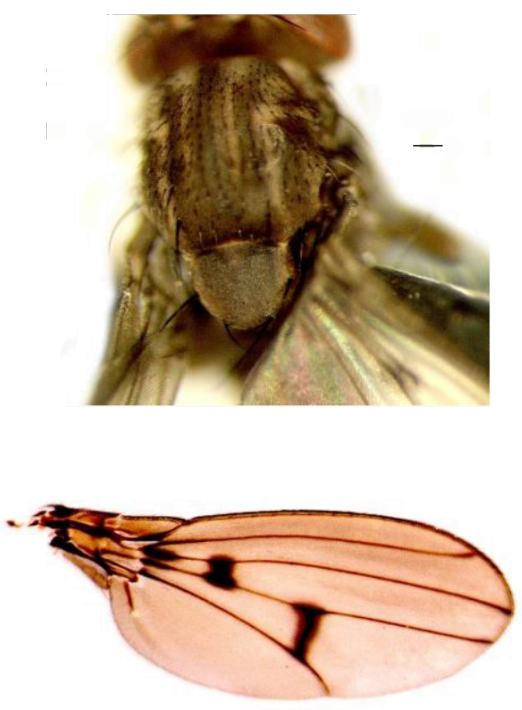

15

Figs 8-15. Morfología externa de D. valenteae sp. nov. y D. griseolineata Duda, 1927. Drosophila valenteae sp. nov., paratipo $\odot: 8$, vista lateral del cuerpo; 9, tórax, vista dorsal; 10, ala derecha; 11, abdomen, vista dorsal. Drosophila griseolineata + (espécimen proveniente de Porto Alegre, Brasil): 12, vista lateral del cuerpo; 13, tórax, vista dorsal ; 14, ala derecha; 15, abdomen, vista dorsal. (Escala: $0.5 \mathrm{~mm}$ ). 


\section{6}

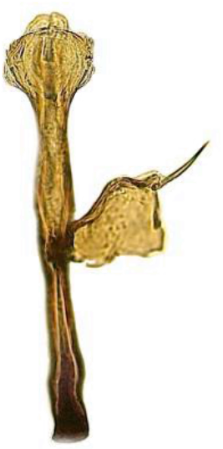

17

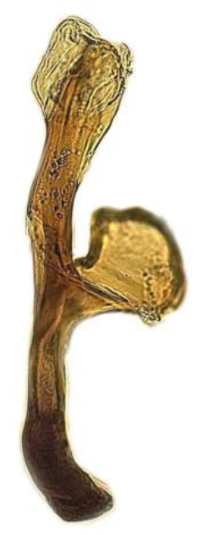

18

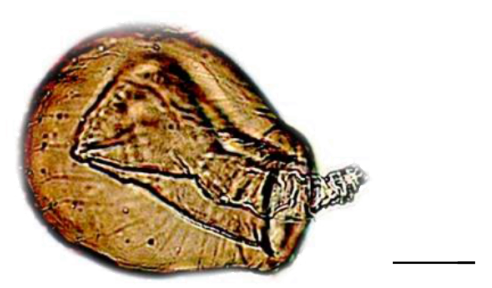

19

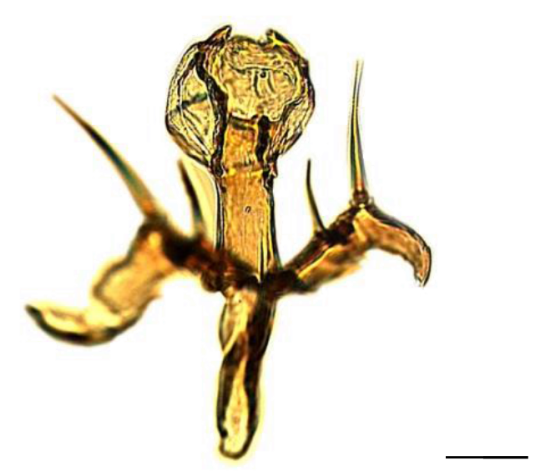

20

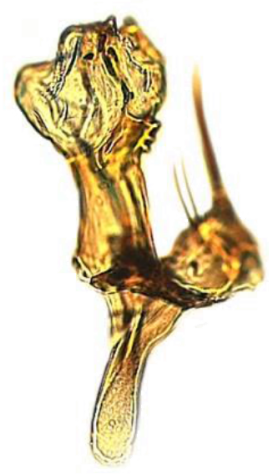

21

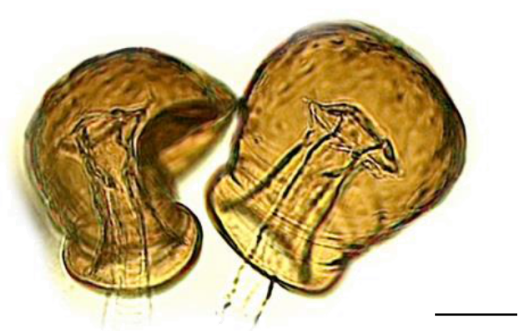

Figs 16-21. Genitalia de D. valenteae sp. nov. y D. griseolineata Duda, 1927. Drosophila valenteae sp. nov., paratipo đo, edeago y paráfisis: 16 , vista ventral; 17, vista lateral; 18, paratipo + , espermateca. Drosophila griseolineata Duda, 1927, §̂, edeago, paráfisis y gonopodio: 19, vista ventral; 20, vista lateral; 21, + , espermateca. (Escala: $0.05 \mathrm{~mm}$ ).

$0.81(0.73-0.85)$. Índice genal 3.90 (3.50-4.70). Índice del ojo $1.15(1.09-1.23)$.

Tórax (Fig. 9). Longitud 1.24 (1.16-1.32) mm. Índice h 1.23 (1.17-1.33). Distancia transversal de las cerdas dorsocentrales $190(183-213) \%$ de la distancia longitudinal. Índice dc 0.62 (0.057-0.69). Distancia entre las cerdas escutelares apicales $69(65-76) \%$ de la distancia entre las cerdas apicales y basales. Índice escutelar 1.24 (1.21-1.30); índice esterno 0.52 (0.48-0.57).

Abdomen. Similar al del macho (Fig. 11).

Alas. Color beige, longitud $2.90(2.75-2.95) \mathrm{mm}$ (Fig. 11). Índices alares 2.41 (2.36-2.69); $C=4.57$ (4.46-4.73), $\mathrm{ac}=2.1(1.95-2.35), \mathrm{hb}=0.29(0.21-0.35), 4 \mathrm{c}=0.52(0.48-0.59)$, $4 \mathrm{v}=1.37(1.30-1.44), 5 \mathrm{x}=1.23(1.17-1.129), \mathrm{M}=0.41(0.37-$ $0.47)$ y prox. $\mathrm{x}=0.45(0.39-0.51)$.

Genitalia. Oviscapto alargado, poco quitinizado y apicalmente redondeado con 14 ovisensillas marginales con punta afilada y cuatro con punta redondeada; seis ovisensillas discales como dientes; tres ovisensillas internas como tricomas y una subterminal, larga y ligeramente curvada (Fig. 7). Espermateca poco quitinizada en forma de globo con invaginación que alcanza $2 / 3$ partes, presenta estrías en la parte externa (Figs 6, 18). 
Etimología. El nombre específico de Drosophila valenteae es en honor a la Dra. Vera Lúcia da Silva Valente profesora de la Universidad Federal Rio Grande do Sul, Brasil, quien ha hecho grandes contribuciones al conocimiento de las drosófilas.

Distribución. Esta especie ha sido capturada solo en la localidad tipo (Cashapamba, Pichincha).

Relación morfológica con otras especies. Drosophila valenteae sp. nov. es similar a nivel de la morfología externa, morfología del edeago y espermateca con Drosophila griseolineata, por lo tanto estas especies estarían emparentadas (Figs 8-15, 16-21). También comparte algunas características de la morfología externa con Drosophila maculifrons Duda, 1927.

\section{Drosophila cumanda sp. nov.}

(Figs 22-31)

urn:lsid:zoobank.org:act:76CC9D4C-EEAD-4E07-A584BFE1CBCB8AEB

Material tipo. Holotipo $\hat{o}^{\lambda}$ (montado en seco, genitalia en microtubo), Ecuador, Napo, Cumandá ( $0^{\circ} 27^{\prime} 13.2^{\prime \prime}$, 7752'46.8”'W, 1864 msnm), III.2014, M. L. Figuero col., L. M. Llangarí \& V. Rafael det. (QCAZI 3162); alotipo $ᄋ$ (montado en seco, genitalia en microtubo), los mismos datos del holotipo (QCAZI 3166); paratipos: 3 $3 \hat{3}, 3 q$ (montados en seco, genitalia en microtubo), los mismos datos del holotipo (QCAZI 3163-3165, 3167-3169).

Diagnosis. Frente marrón con manchas amarillas. Placa orbital marrón con áreas amarillas; cerdas emergiendo de puntos oscuros. Tórax amarillo en el dorso y marrón oscuro en la parte lateral con cerdas y pelos emergiendo de puntos oscuros, algunas veces fusionados. Con cerdas preescutelares. Alas con la vena transversal radial-media (R-M) y la vena transversal media discal-cubital (dM-Cu) sombreadas. Primera sección costal con una hilera de dientes, parte apical de la primera sección costal es oscura (costal lappet). La vena radial $\mathrm{R}_{4+5} \mathrm{y}$ vena media $(\mathrm{M})$ convergentes.
Edeago quitinizado, alargado, ligeramente bífido con la punta quitinizada dirigida hacia la parte dorsal. Región dorsal del edeago con un par de proyecciones como alas, con abertura dorsal.

Descripción del macho, holotipo y paratipos (individuos provenientes de isolínea conservados en alcohol). Morfología externa. Longitud total (cuerpo + alas) 5.45 (4.905.45) mm. Color del cuerpo marrón oscuro (Fig. 23).

Cabeza. Frente marrón con manchas amarillas. Longitud frontal $0.48(0.45-0.58) \mathrm{mm}$. Índice frontal 0.85 (0.82-0.87). Triángulo frontal marrón con la parte central amarilla. Triángulo ocelar marrón amarillento, cerca del 55 (56-61) \% de la longitud, ocelos amarillos. Placa orbital marrón con áreas amarillas; cerdas emergiendo de puntos oscuros, cerda orbital media muy cerca de la orbital anterior, distancia de or3 a or1, 92 (81-92) \% de or3 a vtm, radio or1/or3 0.96 (0.72-0.93), radio or2/or1 $0.74(0.63-0.78)$, cerda postocelar $55(58-63) \%$ y cerda ocelar $71(68-85)$ $\%$ de la longitud frontal; índice vt 0.76 (0.69-0.80); índice vibrisal 0.66 (0.58-0.72). Frontal vitta marrón con áreas amarillas. Gena y postgena marrón. Índice genal 6.30 (5.836.30). Sin subvibrisa. Carina amarilla, prominente y surcada. Proboscis amarilla. Ojos de color rojo oscuro; índice del ojo 1.78 (1.69-1.95). Arista plumosa con cuatro ramas dorsales, dos ventrales, más la terminal bifurcada.

Tórax. Tórax amarillo en el dorso y marrón oscuro en la parte lateral con cerdas y pelos emergiendo de puntos oscuros los cuales algunas veces están fusionados (Fig. 23), longitud 1.61 (1.56-1.69) mm. Índice h 0.76 (0.630.79). Ocho hileras de pelos acrosticales entre las cerdas dorsocentrales anteriores. Distancia transversal de las cerdas dorsocentrales $216(211-255) \%$ de la distancia longitudinal; índice de 0.76 (0.66-0.87). Escutelo marrón oscuro con áreas claras. Un par de cerdas preescutelares. Distancia entre las cerdas escutelares apicales $86(77-94) \%$ de la distancia entre las cerdas apicales y basales; cerdas escutelares basales paralelas. Índice escutelar 0.88 (0.86-0.93). Cerda esternopleural media $1 / 5$ de la cerda esternopleural anterior. Índice esterno 0.87 (0.78-0.92). Patas con fémur y tibia marrón oscuro, los segmentos tarsales marrón amarillentos.
22

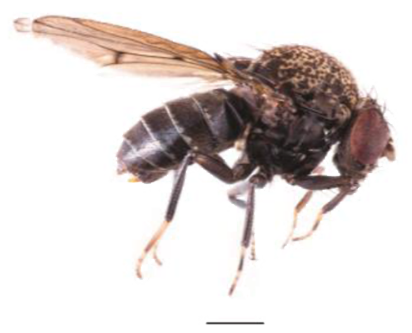

23

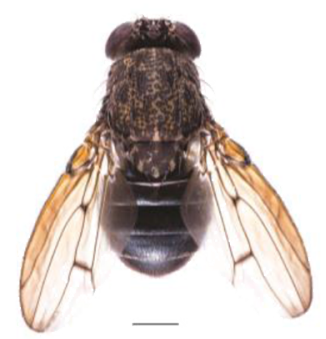

\section{4}

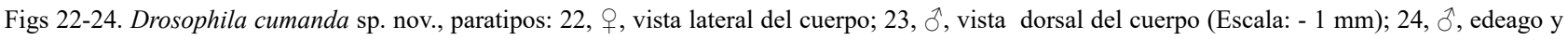
parafisis, vista lateral. (Escala: $0.05 \mathrm{~mm}$ ). 

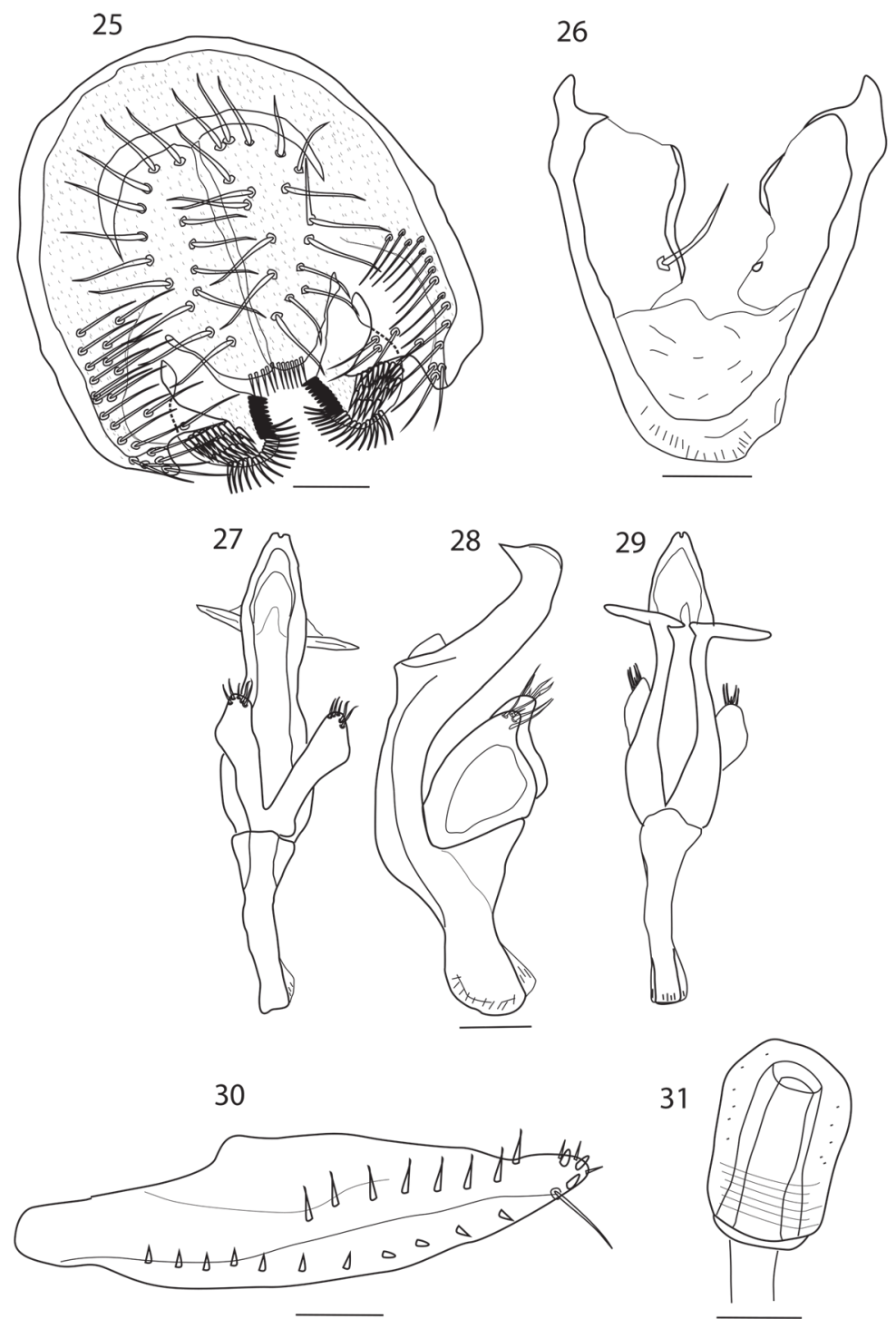

Figs 25-31. Drosophila cumanda sp. nov., holotipo $\widehat{o}: 25$, epandrio; 26, hipandrio y gonopodio; 27-29, edeago y paráfisis, vistas ventral, lateral y dorsal, respectivamente. Alotipo ?: 30, oviscapto; 31, espermateca. (Escala: $0.1 \mathrm{~mm}$ ).

Alas. Marrón claro con la vena transversal radial-media (R-M) y la vena transversal media discal-cubital (dM-Cu) sombreadas. Primera sección costal con una hilera de dientes, parte apical de la primera sección costal es oscura (costal lappet). La vena radial $\mathrm{R}_{4+5} \mathrm{y}$ vena media $(\mathrm{M})$ convergentes (Fig. 23). Longitud del ala 3.97 (3.81-4.01) $\mathrm{mm}$. Índices alares 2.06 (2.10-2.41); $\mathrm{C}=3.01(3.12-3.40)$, $\mathrm{ac}=2.46(2.22$ 2.81), $\mathrm{hb}=0.60(0.57-0.81), 4 \mathrm{c}=0.83(0.78-0.93), 4 \mathrm{v}=1.73$ (1.75-1.82), $5 \mathrm{x}=1.07(1.07-1.16), \mathrm{M}=0.45(0.40-0.47) \mathrm{y}$ prox. $\mathrm{x}=0.85(0.78-0.89)$.

Abdomen. Marrón oscuro. Del primer tergito al sexto tergito pigmentados de color marrón oscuro casi en su totalidad, con una línea blanca e irregular a lo largo del borde posterior de los seis tergitos (Fig. 23).

Genitalia externa. Cerci parcialmente unida al epandrio. Epandrio en la parte dorsal microtricoso, sin cerdas, parte baja del epandrio con 25 cerdas y una proyección triangular y quitinizada sobre el surestilo. Surestilo ovalado, quitinizado, microtricoso, con 9 dientes primarios en el lado derecho y 10 en el izquierdo; no hay diferencia entre las cerdas marginales y dientes secundarios, 25 dientes secundarios y 15 marginales en ambos lados (Fig. 25).

Genitalia interna. Hipandrio en forma "V" con el borde quitinizado, sin arco dorsal, gonopodio quitinizado con una cerda larga (Fig. 26). Edeago quitinizado, en vista ventral y dorsal el extremo distal presenta un surco y termina en una punta quitinizada dirigida hacia la parte dorsal. Región dorsal del edeago con abertura dorsal y un par de proyecciones como alas. Apodema ancho y quitinizado. Rama ventral triangular, paráfisis ovalada con cuatro cerdas largas y una pequeña (Figs 24, 27-29).

Descripción de la hembra, alotipo y paratipos (individuos provenientes de isolínea conservados en alcohol). Morfología externa. Longitud total (cuerpo + 
alas) $5.30(5.05-5.38) \mathrm{mm}$. Color del cuerpo marrón oscuro (Fig. 22).

Medidas hembra Cabeza. Longitud frontal 0.52 (0.48$0.59) \mathrm{mm}$. Índice frontal $0.96(0.88-1.02)$. Triángulo ocelar, cerca del $57(49-64) \%$ de la longitud frontal. Distancia de or3 a or $1,62(62-76) \%$ de or3 a vtm, radio or1/or3 $0.90(0.77-0.88)$, radio or 2 /or $10.35(0.33-0.47)$, cerda postocelar 48 (50-62) $\%$ y cerda ocelar $82(80-91) \%$ de la longitud frontal; índice vt 0.94 (0.92-1.03); índice vibrisal 0.84 (0.78-0.93). Índice genal 5.11 (4.72-5.09). Índice del ojo 2.00 (1.92-1.98).

Tórax (Fig. 22). Longitud 1.65 (1.42-1.65) mm. Índice h 1.27 (1.05-1.35). Distancia transversal de las cerdas dorsocentrales $275(271-280) \%$ de la distancia longitudinal. Índice dc 0.98 (0.85-0.98). Distancia entre las cerdas escutelares apicales 96(89-101)\% de la distancia entre las cerdas apicales y basales; índice escutelar 0.85 (0.76-0.92); índice esterno 1.45 (1.12-1.46).

Alas (Fig. 22). Longitud 4.15 (4.08-4.55) $\mathrm{mm}$. Índices alares $2.32(2.29-2.35) ; \mathrm{C}=3.23$ (3.18-3.46), $\mathrm{ac}=2.54(2.37-2.75), \mathrm{hb}=0.64(0.59-0.66), 4 \mathrm{c}=0.85(0.81-0.90)$, $4 \mathrm{v}=1.77$ (1.65-1.83), $5 \mathrm{x}=1.10(0.96-1.20), \mathrm{M}=0.48$ (0.41$0.54)$ y prox. $\mathrm{x}=0.82(0.79-0.93)$.

Abdomen. Similar al macho (Fig. 22).

Genitalia. Oviscapto quitinizado y apicalmente redondeado con 11 ovisensillas marginales como dientes y tres ovisencillas marginales con punta redondeada; ocho ovisensillas discales como dientes; tres ovisensillas internas como tricomas y una subterminal, larga y ligeramente curvada (Fig. 30). Espermateca ligeramente quitinizada invaginación alcanza casi el borde superior, con estrías circulares en la parte inferior (Fig. 31).

Etimología. Esta especie es nombrada Drosophila cumanda en honor al pueblo de Cumandá cercano al lugar de colecta, provincia de Napo, Ecuador.

Distribución. Esta especie ha sido registrada únicamente en el bosque nublado de los andes orientales de la localidad tipo (Cumandá, Napo).
Ecología. Esta especie emergió con la especie $D$. aracataca del cebo de banano y levadura, utilizado en el campo para la captura de las moscas.

Relación morfológica con otras especies. Drosophila cumanda sp. nov. pertenece al grupo D. annulimana. Esta especie presenta características morfológicas comunes con D. aracataca, D. annulimana, D. araicas y Drosophila aragua Vilela \& Pereira, 1982.

\section{Especies no agrupadas del subgénero $D$. (Drosophila) Drosophila cruzloma sp. nov.}

(Figs 32-40)

urn:lsid:zoobank.org:act:DF7B0C12-8E81-4938-BCF1-
5413031F8A9E

Material tipo. Holotipo $\widehat{\jmath}$ (montado en seco, genitalia en microtubo), Ecuador, Pichincha, Teleférico ( $0^{\circ} 1$ '19' $\mathrm{S}$, 78³1'25.1'W, 3100 msnm), III.2014, M. L. Figuero col., L. M. Llangarí \& V. Rafael det. (QCAZI 3153); alotipo 우 (montado en seco, genitalia en microtubo), los mismos datos del holotipo (QCAZI 3157); Paratipos: $3 \hat{\jmath}, 4$ + (montados en seco, genitalia en microtubo), los mismos datos del holotipo (QCAZI 3154-3156, 3158- 3161).

Diagnosis. Color del cuerpo marrón amarillento. Arista plumosa con cinco ramas dorsales, tres ventrales, más la terminal bifurcada. Seis hileras de pelos acrosticales entre las cerdas dorsocentrales anteriores. Alas marrón con una sombra café en la vena transversal radial-media (R-M), en la vena transversal media discal-cubital (dM-Cu), y en la parte apical de la vena radial $R_{2+3}$, vena radial $R_{4+5}$ y vena media (M). Parte distal del edeago membranosa y ensanchada, lado ventral con dos espolones subapicales dirigidos hacia el centro. En vista lateral estos espolones subapicales tienen la apariencia de dos ganchos con borde aserrado.
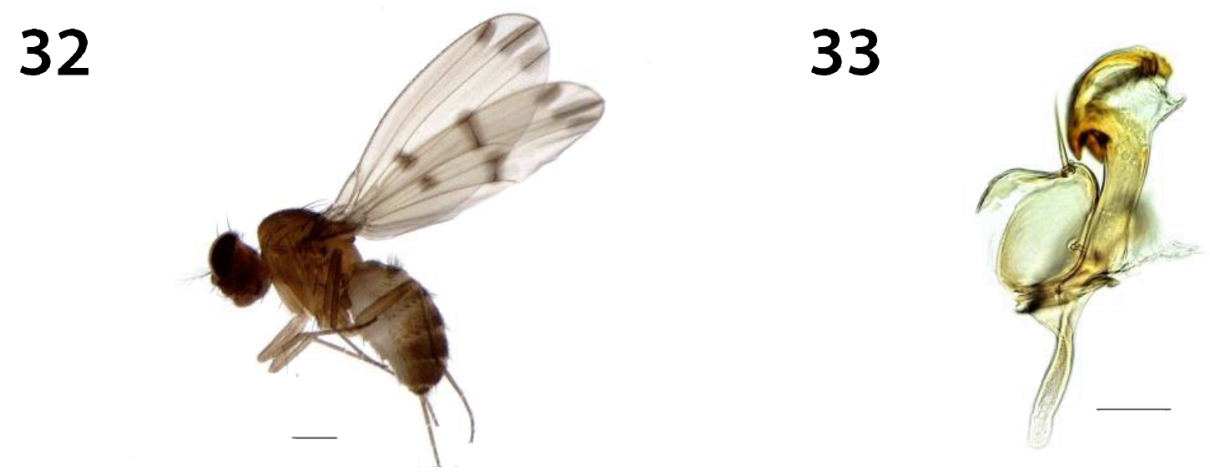

Figs 32, 33. Drosophila cruzloma sp. nov., paratipos $\precsim$ : 32, vista lateral (Escala: $0.5 \mathrm{~mm}$ ); 33, edeago y paráfisis, vista lateral. (Escala: $0.05 \mathrm{~mm}$ ). 

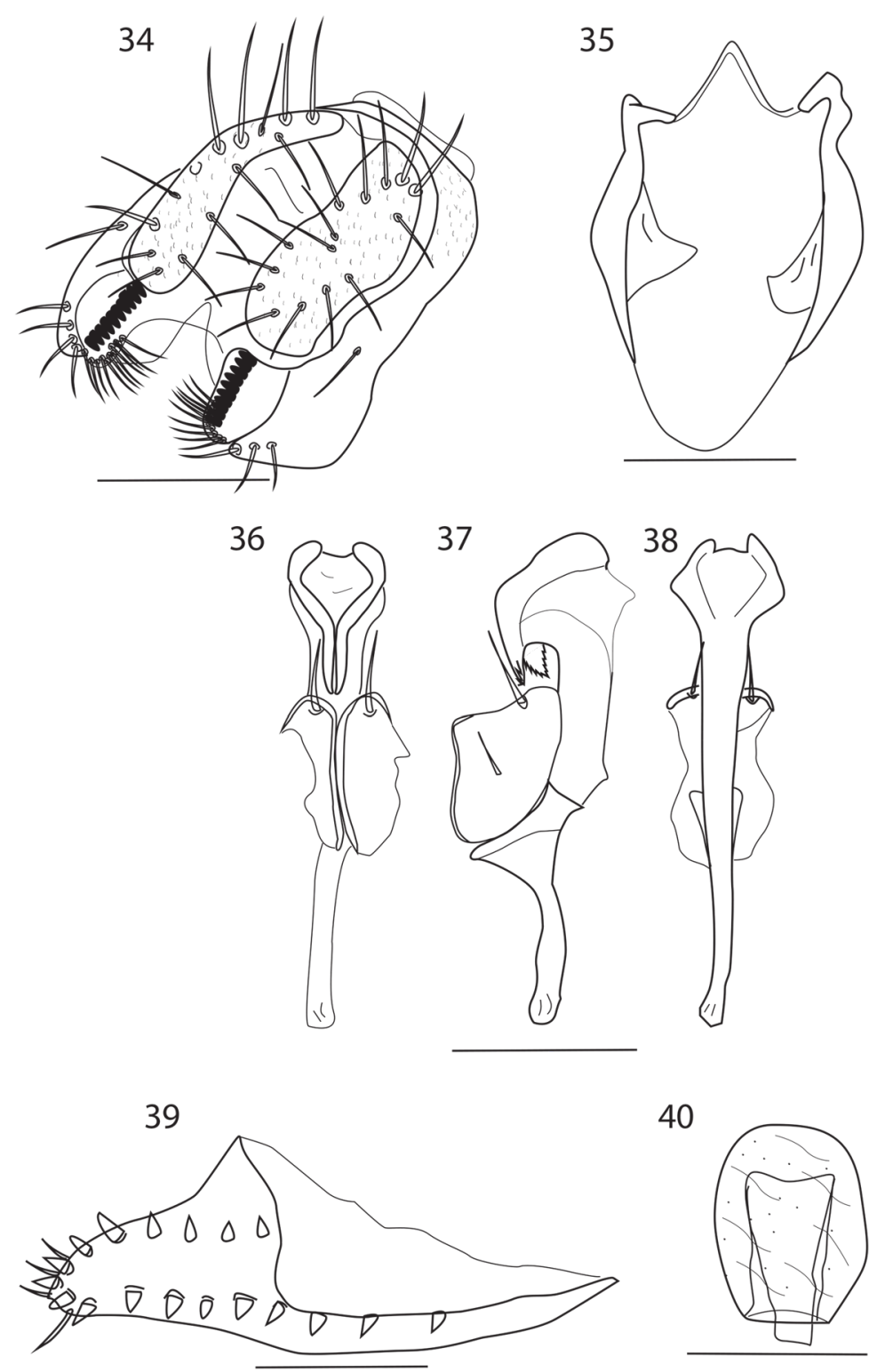

40

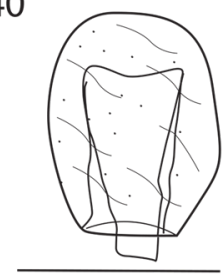

Figs 34-40. Drosophila cruzloma sp. nov., holotipo ổ: 34, epandrio; 35, hipandrio y gonopodio; 36-38, edeago y paráfisis, vistas ventral, lateral y dorsal, respectivamente. Alotipo +: 39, oviscapto; 40, espermateca. (Escala: $0.1 \mathrm{~mm}$ ).

Descripción del macho, holotipo y paratipos (individuos provenientes de isolínea conservados en alcohol). Morfología externa. Longitud total (cuerpo + alas) 4.56 (4.354.60) mm. Color del cuerpo marrón amarillento (Fig. 32).

Cabeza. Frente amarillo polinoso. Longitud frontal 0.35 (0.32-0.42) mm. Índice frontal 0.94 (0.89-0.97). Triángulo frontal marrón amarillento. Triángulo ocelar marrón, cerca del 43(40-46)\% de la longitud, ocelos amarillos. Placa orbital marrón amarillenta; la cerda orbital media cerca de la placa orbital, distancia de or3 a or1, 69(63-72)\% de or3 a vtm, radio or1/or3 0.67 (0.61-0.75), radio or2/or1 $0.38(0.33-0.42)$, cerda postocelar $71(64-78) \%$ y cerda ocelar $85(79-91) \%$ de la longitud frontal; índice vt 1.12 (1.011.22); índice vibrisal 0.90 (0.83-0.94). Frontal vitta amarilla polinosa. Gena y postgena amarillas; índice genal 4.25 (3.98-
4.39). Sin subvibrisa. Carina amarillo polinoso, ligeramente prominente, no surcada. Proboscis amarilla. Ojos de color rojo vinoso. Índice del ojo 1.28 (1.19-1.35). Segundo y tercer segmento antenal marrón amarillento. Arista plumosa con cinco ramas dorsales, tres ventrales, más la terminal bifurcada.

Tórax. Marrón amarillento, longitud 1.16 (1.10$1.21) \mathrm{mm}$. Índice h $0.69(0.60-0.75)$. Seis hileras de pelos acrosticales entre las cerdas dorsocentrales anteriores. Distancia transversal de las cerdas dorsocentrales 237(201$243) \%$ de la distancia longitudinal. Índice dc 0.82 (0.76-0.86). Escutelo del mismo color que el tórax, distancia entre las cerdas escutelares apicales $85(78-93) \%$ de la distancia entre las cerdas apicales y basales; índice escutelar 1.28 (1.12-1.38). Cerda esternopleural media $3 / 4$ de la cerda esternopleural anterior; índice esterno 0.60 (0.55-0.68). Patas amarillas. 
Alas. Color marrón con una sombra café en la vena transversal radial-media (R-M), en la vena transversal media discal-cubital ( $\mathrm{dM}-\mathrm{Cu})$, y en la parte apical de la vena radial $\mathrm{R}_{2+3}$, vena radial $\mathrm{R}_{4+5} \mathrm{y}$ vena media (M) (Fig. 32). Longitud del ala $3.11(3.01-3.15) \mathrm{mm}$. Índices alares 2.28 (2.25-2.32); C= $3.94(3.91-3.98), a c=1.80(1.73-1.81), h b=0.30(0.27-0.31)$, $4 \mathrm{c}=0.70(0.68-0.73), 4 \mathrm{v}=1.48(1.45-1.50), 5 \mathrm{x}=1.15(1.12-$ $1.16), \mathrm{M}=0.46(0.44-0.48)$ y prox. $\mathrm{x}=0.91$ (0.89-0.93).

Abdomen. Marrón amarillento, con línea media dorsal. Del primer al quinto tergito con una franja oscura que ocupa casi todo el tergito y que se adelgaza hacia los lados; sexto tergito oscuro.

Genitalia externa. Cerci libre microtricosa. Epandrio en la parte dorsal microtricoso, sin cerdas, parte baja del epandrio no microtricoso, lóbulo ventral en forma de dedo con tres cerdas. Surestilo no microtricoso, lado derecho e izquierdo con diez (9-10) dientes primarios; sin dientes secundarios; lado derecho e izquierdo con diez cerdas marginales (10-11) (Fig. 34).

Genitalia interna. Hipandrio en forma de escudo con el contorno quitinizado, gonopodio triangular, parte media del arco dorsal ensanchado (Fig. 35). Edeago quitinizado, recto y tubular, parte distal membranosa y ensanchada, lado ventral con dos espolones subapicales dirigidos hacia el centro. En vista lateral estos espolones subapicales tienen la apariencia de dos ganchos con borde aserrado. Rama ventral corta. Apodema poco quitinizado. Paráfisis ovalada con una cerda larga en el extremo distal y una cerda corta en el centro que probablemente corresponde al gonopodio (Figs 33, 36-38).

Descripción de la hembra, alotipo y paratipos (individuos provenientes de isolínea conservados en alcohol). Morfología externa. Longitud total (cuerpo + alas) 4.75 (4.204.88) mm. Color del cuerpo marrón amarillento.

Medidas hembra. Cabeza. Longitud frontal 0.32 (0.28$0.39) \mathrm{mm}$. Índice frontal 0.94 (0.86-0.98). Triángulo ocelar, cerca del $44(39-49) \%$ de la longitud frontal. Distancia de or3 a or1, 66 (62-78) \% de or3 a vtm, radio or1/or3 0.72 (0.67$0.78)$, radio or $2 /$ or1 $0.43(0.39-0.48)$, cerda postocelar $72(70$ $76) \%$ y cerda ocelar $88(81-93) \%$ de la longitud frontal; índice vt 1.11 (1.02-1.31); índice vibrisal 0.89 (0.75-0.94). Índice genal 3.50 (3.22-4.8). Índice del ojo $1.30(1.12-1.50)$.

Tórax. Longitud $1.25(1.15-1.33) \mathrm{mm}$. Índice h 0.83 (0.75-1.05). Distancia transversal de las cerdas dorsocentrales 178 (171-182) \% de la distancia longitudinal. Índice dc 0.65 (0.58-0.71). Distancia entre las cerdas escutelares apicales 76(69-81)\% de la distancia entre las cerdas apicales y basales; índice escutelar 1.33 (1.26-1.41); índice esterno 0.80 (0.68-0.86).

Alas (Fig. 32). Longitud $3.53(3.15-3.70) \mathrm{mm}$. Índices alares $2.46(2.39-2.72) ; \mathrm{C}=3.81(3.65-4.01), \mathrm{ac}=$ $1.74(1.67-1.85), \mathrm{hb}=0.36(0.29-0.43), 4 \mathrm{c}=0.78(0.71-0.88)$, $4 \mathrm{v}=1.62(1.57-1.74), 5 \mathrm{x}=1.03(0.98-1.16), \mathrm{M}=0.47(0.39$ $0.53)$ y prox. $\mathrm{x}=0.90(0.83-0.96)$.

Abdomen. Marrón, con línea media dorsal. Del primer al cuarto tergito con una franja oscura en el borde posterior que se adelgaza a los lados; quinto y sexto tergito con una línea oscura en el borde posterior.

Genitalia. Oviscapto ensanchado, poco quitinizado y apicalmente redondeado con 14 ovisensillas marginales como dientes; cuatro ovisensillas discales como dientes; tres ovisensillas internas como tricomas y una subterminal, larga y ligeramente curvada (Fig. 39). Espermateca poco quitinizada alargada y con estrías diagonales y puntos refringentes, invaginación ensanchada que alcanza $3 / 4$ partes de la longitud de la espermateca (Fig. 40).

Etimología. El nombre Drosophila cruzloma sp. nov. se debe a la montaña Cruz Loma ubicada en el lado occidental de la ciudad de Quito (cerca del teleférico), lugar de colecta.

Distribución. Peñas Blancas (bosque altoandino de Polylepis) $\left(0^{\circ} 17^{\prime} 56.1^{\prime \prime} \mathrm{S}, 78^{\circ} 14^{\prime} 37.5^{\prime \prime} \mathrm{W}\right)$ vía a Papallacta. Parque Arqueológico Rumipamba $\left(0^{\circ} 10^{\prime} 49.9\right.$ 'S, $78^{\circ} 30^{\prime} 04.8^{\circ} \mathrm{W}$ ) y cercanías del teleférico de Quito.

\section{Drosophila tinalandia sp. nov.}

(Figs 41-50)

urn:1sid:zoobank.org:act:0A66E0BD-FD8B-421A-BEB0$756723 \mathrm{~A} 0126 \mathrm{C}$

Material tipo. Holotipo ô (montado en seco, genitalia en microtubo), Ecuador, Santo Domingo de los Colorados,
41

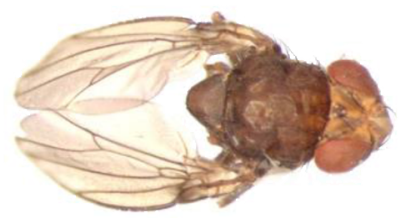

42

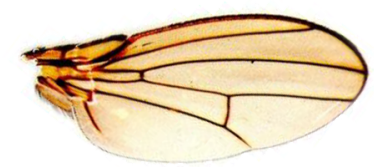

43

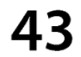

Figs 41-43. Drosophila tinalandia sp. nov., paratipos: 41, + , tórax, vista dorsal (espécimen seco); 42,, , ala derecha (Escala: - 0.5 mm); 43, 3 , edeago y paráfisis, vista lateral. (Escala: $0.05 \mathrm{~mm}$ ). 

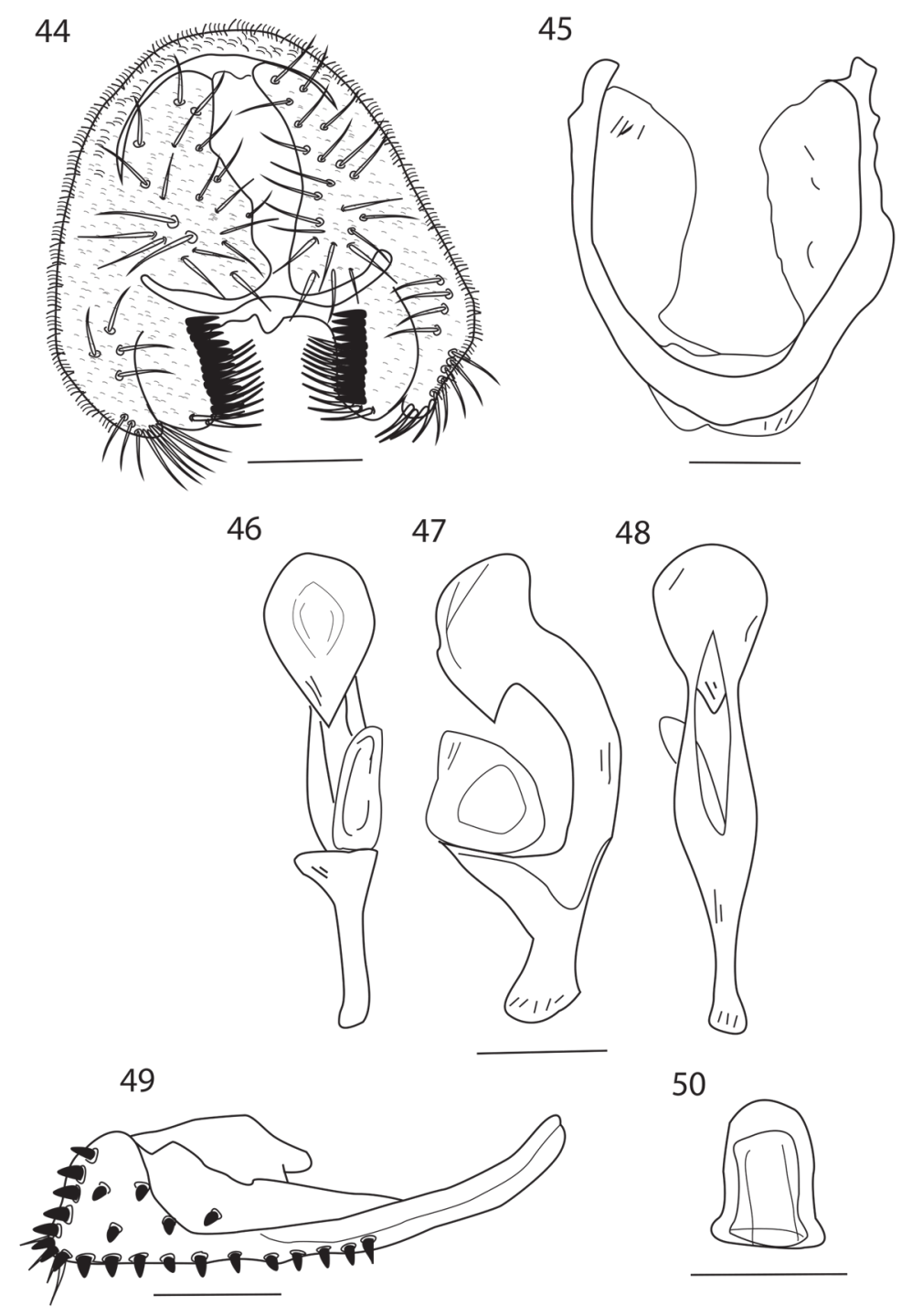

Figs 44-50. Drosophila tinalandia sp. nov., holotipo 37 : 44, epandrio; 45, hipandrio y gonopodio; 46-48, edeago y paráfisis, vistas ventral, lateral y dorsal, respectivamente; Alotipo $\odot:$ 49, oviscapto; 50; espermateca. (Escala: $0.1 \mathrm{~mm}$ ).

Tinalandia $\left(0^{\circ} 18^{\prime} 34^{\prime \prime} \mathrm{S}, 7^{\circ} 03^{\prime} 00^{\prime} \mathrm{W}, 660 \mathrm{msnm}\right)$, VII.2013, V. Rafael col., L. M. Llangarí \& V. Rafael det. (QCAZI 3131); alotipo + (montado en seco, genitalia en microtubo), los mismos datos del holotipo (QCAZI 3130); paratipos: $\widehat{O}^{\text {, }}$ q (montados en seco, genitalia en microtubo), los mismos datos del holotipo (QCAZI 3132, 3129).

Diagnosis. Cuerpo marrón. Arista plumosa con ocho ramas dorsales, tres ventrales. Tórax con una banda más clara en el centro y con ocho hileras de pelos acrosticales entre las dorsocentrales anteriores. Cerdas escutelares basales paralelas. Cerda esternopleural media $1 / 5$ de la cerda esternopleural anterior. Epandrio y cerci microtricosos. Edeago quitinizado curvado hacia la parte ventral, parte distal ensanchado, parte dorsal redondeado con la parte ventral que termina en punta.

Descripción del macho, holotipo (individuo conservado en alcohol). Morfología externa. Longitud total (cuerpo + alas) del holotipo $5.11 \mathrm{~mm}$. Color del cuerpo marrón.

Cabeza. Frente marrón amarillenta (Fig. 41). Longitud frontal $0.47 \mathrm{~mm}$. Índice frontal 0.87 . Triángulo frontal amarillo polinoso. Triángulo ocelar marrón oscuro, cerca del $45 \%$ de la longitud, ocelos amarillos polinosos. Placa orbital marrón amarillenta; cerda orbital media cerca de la orbital anterior y hacia el borde exterior de la placa orbital, distancia de or 3 a or $1,63 \%$ de or 3 a vtm, radio or $1 /$ or 30.82 , radio or $2 /$ or 10.48 , cerda postocelar $68 \%$ y cerda ocelar $81 \%$ de la longitud frontal; índice vt 1.38 . Índice vibrisal 0.83 . Frontal vitta, gena y postgena amarillas polinosas. Índice genal 5.20. Una cerda oral bien desarrollada. Carina amarilla polinosa, prominente, no surcada. Proboscis y palpos amarillo oscuro. Ojos de color rojo vinoso, índice del ojo 1.3. Arista plumosa con ocho ramas dorsales, tres ventrales, más la terminal bifurcada. 
Tórax. Marrón polinoso (dorado) con una banda más clara a lo largo de las cerdas dorsocentrales y en la parte media del tórax, longitud $1.71 \mathrm{~mm}$ (Fig. 41). Índice h 1.13. Ocho hileras de pelos acrosticales entre las cerdas dorsocentrales anteriores. Distancia transversal de las cerdas dorsocentrales $266 \%$ de la distancia longitudinal. Índice dc 0.73. Escutelo del mismo color que el tórax, distancia entre las cerdas escutelares apicales $58 \%$ de la distancia entre las cerdas apicales y basales. Cerdas escutelares basales paralelas; índice escutelar 0.83 . Cerda esternopleural media $1 / 5$ de la esternopleural anterior; índice esterno 0.84 . Patas con el fémur y la tibia marrón, segmentos tarsales marrón amarillento.

Alas. Marrón amarillento (Fig. 42). Longitud del ala $3.27 \mathrm{~mm}$. Índices alares 2.27; $\mathrm{C}=2.66, \mathrm{ac}=2.17, \mathrm{hb}=0.77$, $4 \mathrm{c}=0.80,4 \mathrm{v}=1.53,5 \mathrm{x}=1.08, \mathrm{M}=0.40$ y prox. $\mathrm{x}=0.43$.

Abdomen. Color marrón. Primer tergito marrón amarillento. Del segundo al cuarto tergito con línea media dorsal. Quinto y sexto tergito marrón.

Genitalia externa. Epandrio extremadamente microtricoso. Cerci microtricoso y parcialmente unidas al epandrio. Parte baja del epandrio con trece cerdas. Surestilo microtricoso con 12 dientes primarios y 11-12 cerdas marginales en cada lado (Fig. 44).

Genitalia interna. Hipandrio ligeramente más pequeño que el epandrio, y en forma de escudo con el contorno quitinizado, gonopodio voluminoso (Fig. 45). Edeago quitinizado, ápice ensanchado, redondeado, curvado hacia la parte ventral y terminando en punta. Paráfisis triangular. Rama ventral bien desarrollada. Apodema más corto que el edeago y lateralmente ancho (Figs 43, 46-48).

Descripción de la hembra, alotipo (individuo descrito en vivo). Morfología externa. Longitud total (cuerpo + alas) del alotipo $4.84 \mathrm{~mm}$. Color del cuerpo marrón.

Medidas e índices hembra: Cabeza. Longitud frontal $0.46 \mathrm{~mm}$. Índice frontal 0.88 . Triángulo ocelar cerca del $39 \%$ de la longitud frontal. Distancia de or 3 a or $1,57 \%$ de or3 a vtm, radio or $1 /$ or 30.80 , radio or2/or 10.48 , cerda postocelar $59 \%$ y cerda ocelar $83 \%$ de la longitud frontal; índice vt 0.90; índice vibrisal 0.84. Índice genal 5.06. Índice del ojo 1.15 .

Tórax. Longitud $1.45 \mathrm{~mm}$. Índice h 1.22. Distancia transversal de las cerdas dorsocentrales $245 \%$ de la distancia longitudinal. Índice dc 0.73 . Distancia entre las cerdas escutelares apicales $57 \%$ de la distancia entre las cerdas apicales y basales; índice escutelar 0.95 ; índice esterno 0.86 .

Alas. Longitud $3.04 \mathrm{~mm}$. Índice alar 2.17; $\mathrm{C}=2.65$, $\mathrm{ac}=2.25, \mathrm{hb}=0.67,4 \mathrm{c}=0.80,4 \mathrm{v}=1.42,5 \mathrm{x}=1.12, \mathrm{M}=0.41 \mathrm{y}$ prox. $\mathrm{x}=0.46$.

Genitalia. Oviscapto quitinizado, alargado, apicalmente en punta con 19 ovisensillas marginales con punta redondeada; cinco ovisensillas discales con punta redondea; tres ovisencillas pequeñas como tricomas y una ovisencilla más larga (Fig. 49). Espermateca ligeramente quitinizada en forma de campana con invaginación que alcanza 2/3 partes (Fig. 50).

Etimología. El nombre de Drosophila tinalandia sp. nov. hace referencia al lugar de captura, Tinalandia es una localidad de la provincia de Santo Domingo de los Tsáchilas.

Distribución. Esta especie ha sido registrada en el bosque nublado de la localidad tipo (Tinalandia, Santo Domingo de los Tsáchilas).

Ecología. Esta especie fue recolectada directamente de las inflorescencias de Xanthosoma spp. (Araceae).

\section{DISCUSIÓN}

Drosophila valenteae sp. nov. fue ubicada en el grupo D. guarani porque comparte algunas similitudes con $D$. griseolineata a nivel de la morfología externa, y la genitalia de machos y hembras. Ambas especies presentan seis hileras de pelos acrosticales entre las dorsocentrales anteriores, alas con las venas transversales sombreadas, edeago recto y tubular, con la parte distal ensanchada, membranosos y con microproyecciones a modo de espina, además la espermateca es poco quitinizada en forma de globo y con una profunda invaginación. Las diferencias entre estas dos especies están en la parte apical del edeago de $D$. valenteae sp. nov. que tiene solo dientes conspicuos, mientras que $D$. griseolineata en la base de la cabeza del edeago presenta dos estructuras pequeñas dentadas y quitinizadas que se continúan con otros dientes conspicuos hacia la parte apical. Otra diferencia en $D$. valenteae sp. nov. es la presencia de una membrana dorsal con puntos brillantes que sobrepasa la parte dorsal del edeago mientras que en $D$. griseolineata no sobrepasa el ápice del edeago. Por otro lado, existen diferencias también en la pigmentación de las alas; $D$. valenteae sp. nov. tiene la vena transversal radial-media (R-M) y la vena transversal media discal-cubital (dM-Cu) ligeramente sombreadas (Fig. 10), y en D. griseolineata las alas presentan manchas conspicuas sobre ambas venas transversales y cerca de la bifurcación basal de las venas $\mathrm{R}$, además la punta de $\mathrm{R}_{2+3}$ ligeramente sombreada (VIlela \& Bächli, 1990). Así mismo, el tórax de $D$. valenteae sp. nov. es de color marrón uniforme, pero en el tórax de $D$. griseolineata se observa varias franjas claras. Otra especie del grupo D. guarani con algunas características similares es $D$. maculifrons, las dos especies presentan alas sombreadas a lo largo de las venas transversales, la arista de $D$. valenteae al igual que la de $D$. maculifrons es plumosa con cinco ramas dorsales, tres ventrales, más la terminal bifurcada, también ambas especies tienen seis hileras de pelos acrosticales (VILELA \& BäChli, 1990).

Drosophila cumanda sp. nov. ha sido incluida en el grupo D. annulimana porque presenta varios caracteres del grupo. En el tórax las cerdas y pelos emergen de puntos oscuros algunas veces fusionados. También esta especie nueva tiene las alas de color marrón claro con la vena transversal radial-media (R-M) y la vena transversal media discal-cubital 
(dM-Cu) sombreadas. Además la primera sección costal posee una hilera de dientes y presenta convergencia apical en las venas alares $R_{4+5}$ y M (Breuer \& Pavan, 1950). Al igual que las otras especies del grupo, D. cumanda sp. nov. es grande, mide 4.90-5.45 mm. El tamaño de las especies del grupo D. annulimana oscila alrededor de $4.0 \mathrm{~mm}$ (GotTschalk et al., 2012). Además, D. cumanda sp. nov. posee dos cerdas preescutelares, como en $D$. annulimana, $D$. aracataca, D. aragua, D. araicas, D. arauna Pavan \& Nacrur 1950, D. paratarsata Vilela, 1985 y D. tarsata; mientras que D. arapuan da Cunha \& Pavan, 1947, D. arassari da Cunha \& Frota-Pessoa, 1947, D. gibberosa Patterson \& Mainland, 1943, D. ararama Pavan \& da Cunha, 1947, D. breuerae Rocha, 1987, D. caxiuana Gottschalk et al., 2012, D. talamancana Vilela \& Pereira, 1987 y D. schineri Vilela \& Pereira, 1987 no presentan cerdas preescutelares. En la genitalia interna, se puede observar en el ápice del edeago una prominencia rígida, ligeramente bífida dirigida hacia la parte dorsal (igual que en $D$. annulimana, D. araicas, $D$. aracataca y $D$. aragua), también presenta dos procesos laterales como alas en la parte dorsal del edeago (presente en $D$. annulimana, D. araicas, D. aracataca, D. aragua, $D$. arauna, D. paratarsata, D. tarsata, D. breuerae y D. yana). En la paráfisis de las especies de este grupo a excepción de D. yana existe al menos una cerda.

Las especies $D$. cruzloma sp. nov. y D. tinalandia sp. nov. pertenecen al subgénero Drosophila, no fueron asignadas a ningún grupo de especies ya que no poseen las suficientes características para poder agruparlas. Sin embargo, la presencia de arco dorsal en D. cruzloma sp. nov sugiere que podría estar relacionada con las especies de la radiación inmigrans-tripunctata. Mientras que $D$. tinalandia sp. nov. por la morfología de la genitalia del macho estaría relacionada a la radiación virilis-repleta (MARKow \& O'GradY, 2007), y por su lugar de colecta, sobre la inflorescencia de Xanthosoma spp. podría tratarse de una especie antofílica.

Agradecimientos. A la Pontificia Universidad Católica del Ecuador por financiar los proyectos No. J13054 y K13051. Al Ministerio del Ambiente por otorgar el permiso de colección $\mathrm{N}^{\circ}$ 001-10 IC-FAUDNB/ MA y a la Doctora Vera Valente (Universidad Federal Rio Grande do Sul, Brasil), quién nos facilitó una cepa de $D$. griseolineata proveniente de Brasil, para realizar las comparaciones con la nueva especie $D$. valenteae. Así mismo expresamos nuestra gratitud con Anita Peñafiel y Jorge Vela por su colaboración en la toma y edición de fotos.

\section{REFERENCIAS BIBLIOGRÁFICAS}

ACUrio, A. E. \& Rafael, V. L. 2009. Diversity and geographical distribution of Drosophila (Diptera, Drosophilidae) in Ecuador. Drosophila information service 92:20-25.

BäCHLI, G. 2017. TaxoDros: The Database on Taxonomy of Drosophilidae. Available at $<$ http://www.taxodros.unizh.ch $>$. Accessed on 10 November 2017.

Bächli, G.; Vilela, C. R.; Andersson, S. \& Saura, A. 2004. Fauna Entomologica Scandinavica: The Drosophilidae (Diptera) of Fennoscandia and Denmark, vol. 39. Leiden, New York, Ed. Brill. 362p.

Breuer, M. E. \& PAVAn, C. 1950. Genitália masculina de "Drosophila" (Diptera): grupo “annulimana”. Revista Brasileira de Biologia 10:469-488.

Cabezas, M. B. \& Rafael, V. 2013. Una nueva especie del grupo Drosophila annulimana (Diptera, Drosophilidae) y un nuevo registro en las Provincias de Pichincha y Napo, Ecuador. Iheringia, Série Zoologia 103(4):357-360.

CÉSPEDES, D. \& RAFAeL, V. 2012. Cuatro especies nuevas del género Drosphila (Diptera, Drosophilidae) miembros del grupo mesophragmatica en los Andes ecuatorianos. Iheringia, Série Zoologia 102(1):71-79.

Gottschalk, M. S.; Martins, M. B.; Praxedes, C. \& de Medeiros, H. F. 2012. A new Amazonian species from the Drosophila annulimana species group (Diptera, Drosophilidae). Revista Brasileira de Entomologia 56(4):431-435.

Markow, T. A. \& O'Grady, P. M. 2007. Drosophila: A guide to species identification and use. Netherlands, Academic Press. 259p.

Peñafiel-Vinueza, A. D. \& Rafael, V. 2018. Five new species of Drosophila guarani group from the Andes of southern Ecuador (Diptera, Drosophilidae). ZooKeys 781:141-163. https://doi.org/10.3897/ zookeys.781.22841

Rafael, V.; Arcos, G. \& Arcos, L. 2000. Ecología y distribución del género Drosophila en Guayllabamba y el Quinche, Provincia de PichinchaEcuador. Revista de la Pontificia Universidad del Ecuador 65:130-155.

Ratcov, V.; Vilela, C. R. \& GoÑI, B. 2017. A new species of Neotropical Drosophila (Diptera, Drosophilidae) belonging to the guarani group. Revista Brasileira de Entomología 61(3):232-238. http://dx.doi. org/10.1016/j.rbe.2017.06.002

Ross, C. L. \& MARKOw, T. A. 2006. Microsatellite variation among diverging populations of Drosophila mojavensis. Journal of Evolutionary Biology 19:1691-1700.

Tosi, D.; Pereira, M. A. \& Vilela, C. R. 2007. Polytene chromosomes and phylogenetic relationships in ten Drosophila species of the annulimana group (Diptera, Drosophilidae). Genetics and Molecular Biology 30(4):1169-1180.

Vaz, S.C.; Vilela, C. R. \& Carvalho, A.B. 2018. Two new species of Drosophila (Diptera, Drosophilidae) associated with inflorescences of Goeppertia monophylla (Marantaceae) in the city of São Paulo, state of São Paulo, Brazil. Revista Brasileira de Entomología 62(2):159168. https://doi.org/10.1016/j.rbe.2018.03.003

Vela, D. \& Rafael, V. 2004. Three new Andean species of Drosophila (Diptera, Drosophilidae) of the mesophragmatica group. Iheringia, Série Zoologia 94(3):295-299.

Vilela, C. R. \& BäCHLI, G. 1990. Taxonomic studies on Neotropical species on seven genera of Drosophila (Diptera). Mitteilungen der Schweizerischen Entomologischen Gesellschaft 63:1-332. 